\title{
Standardization of reporting urine cytology: the paris system (TPS)
}

\begin{abstract}
The aim of urinary cytology is to detect urothelial carcinoma that is clinically significant, namely high-grade urothelial carcinoma (HGUC). A program was set to standardize urine cytology reporting similar to the Bethesda System for Reporting Cervical Cytology and the Bethesda System for Reporting Thyroid Cytology during the 18th International Congress of Cytology in Paris in May 2013. Eminent cytopathologists, surgical pathologists, and urologists assembled and formed the Paris System Working Group With support from both the International Academy of Cytology and the American Society of Cytopathology, committees were formed to discuss the existing controversies, questions and published literature in the field of urinary tract (UT) cytology, aiming at forming an international consensus on reporting UT specimens. In late 2015, the consensus group published their guidelines, known as The Paris System (TPS) for Reporting Urinary Cytology. TPS consists of 7 diagnostic categories and also tries to define several morphological criteria. This article tries to summarize and highlight essential points of The Paris System.
\end{abstract}

Keywords: the paris system, urine cytology, urothelial carcinoma
Volume 2 Issue I - 2017

\author{
Sara E Khalifa \\ Pathology department, Cairo University, Egypt
}

Correspondence: Sara E Khalifa, Pathology department, faculty of medicine, Cairo university, Egypt,

Email Sara.mekawy@kasralainy.edu.eg

Received: January 06, 2017 | Published: January 25, 2017
Abbreviations: HGUC, high-grade urothelial carcinoma; UT, urinary tract; TPS, the paris system; N/C, nuclear cytoplasmic ratio; SHGUC, suspicious for high grade urothelial carcinoma; LGUN, low grade urothelial neoplasia

\section{Introduction}

For any reporting system to be successful and be applied in daily practice, it must be based on consensus, evidence, inclusion, acceptance, and understanding. ${ }^{1}$ The main goal of urinary cytology is the detection of urothelial carcinoma that is clinically significant, namely highgrade urothelial carcinoma (HGUC). Therefore, the understanding of this disease, and particularly its pathogenesis, was crucial in the process of creating The Paris System for Reporting Urinary Cytology. A program to address standardization of urine cytology reporting similar to the Bethesda System for Reporting Cervical Cytology and the Bethesda System for Reporting Thyroid Cytology was conceived at the 18th International Congress of Cytology in Paris in May 2013 where eminent cytopathologists, surgical pathologists, and urologists assembled and formed the Paris System Working Group With support from both the International Academy of Cytology and the American Society of Cytopathology, committees were formed to discuss the existing controversies, questions and published literature in the field of urinary tract (UT) cytology, aiming at forming an international consensus on reporting UT specimens. In late 2015, the consensus group published their guidelines, known as The Paris System (TPS) for Reporting Urinary Cytology. ${ }^{2}$

\section{Discussion}

TPS categories are as follows:
I. Non-diagnostic or Unsatisfactory.
II. Negative for High Grade Urothelial Carcinoma.
III. Atypia.

IV. Suspicious for High Grade Urothelial Carcinoma.

\section{Low Grade Urothelial Neoplasia (LGUN).}

VI. High Grade Urothelial Carcinoma (HGUC).

VII. Other malignancies, primary and metastatic..$^{3-5}$

\section{Non-diagnostic or unsatisfactory}

It was decided in The Paris System that any sample with atypical, suspicious or malignant cells should be called adequate and reported. So the question of inadequacy comes only when the sample does not show any findings indicative of any disease process. Studies on volume, types of sample, sampling method, and cellularity of urine samples are limited and conclusions are varied. ${ }^{5}$ There is a common misperception that cells in body fluids are solutes in a homogenous solution. However, urine is a heterogeneous mixture of non-solute particulates: crystals, microorganisms, decaying cell remnants, and cells. It is heterogeneous because the particulates are not evenly distributed throughout the volume. Cells are denser than most aqueous solutions, so they sink. If only the supernatant, which is acellular or paucicellular, is examined because the first drops of a urine stream were not captured in the specimen container or because the specimen was inadvertently decanted, the results will be suboptimal. ${ }^{6}$ It has also been stated that if urothelial cells are obscured by any inlammation, blood, mucin, lubricant etc, then the sample should be called inadequate. TPS has proposed that in instrumented specimens, 2600 cells or at least 20 cells in 10 HPF may be taken as adequacy criteria. So when there are 10-20 cells/10 HPF should be reported as "satisfactory but limited by low cellularity" and those with $<10$ cells $/ 10$ HPFs should be under "unsatisfactory/non diagnostic". 5

\section{Negative for high grade urothelial carcinoma}

Because TPS acknowledges that UT cytopathology has a poor sensitivity for detecting LGUN, the benign category does not exclude the presence of LGUN. Instead, the benign category indicates that a specimen does not contain HGUC cells, or any cells with features concerning for HGUC, as defined by the criteria below. A certain number of false-negatives is expected, especially in voided urine 
samples which require tumour cells to be shed into the urine before they can be diagnosed. Serial samples may improve sensitivity for detection.

\section{Atypia (atypical urotelial cells, AUC)}

For a diagnosis of AUC, the sine qua non criterion is high nuclear cytoplasmic ratio $(\mathrm{N} / \mathrm{C})(>0.5)$ in non-superficial and non-degenerated urothelial cells. In addition, one of the following criteria must also be present: mild to moderate nuclear hyperchromasia (compared with a benign urothelial cell or a squamous cell, used as an internal control), irregular nuclear membranes (chromatinic rim or nuclear contour), and irregular, coarse chromatin pattern. To date, patients with a diagnosis of AUC have been managed in a manner similar to patients assigned a "negative" diagnosis. With the refocus by the Paris System on relative risk of all categories predicting HGUC, and clear criteria provided for the diagnosis of AUC, the hope is that the "atypia" rate of urinary cytology will be reduced universally. As this concept becomes more accepted worldwide, a shift in the management strategy of AUC will inevitably follow, perhaps requiring a much closer follow-up and evaluation of patients carrying this diagnosis. ${ }^{5}$

\section{Suspicious for high grade urothelial carcinoma, (SHGUC)}

The suspicious category is meant to be the equivalent of similar categories in other reporting systems, including the terminology 'can not exclude high-grade urothelial carcinoma'. Overall, the suspicious category is meant to imply a high risk of HGUC and used in instances in which an unequivocal diagnosis of HGUC cannot be made as a result of degenerative changes, a suboptimal number of HGUC cells, or atypical cells which do not fulfill all the criteria of the HGUC category.

The criteria for the SHGUC category are:

I. High N/C ratio. Atypical cells should have an N/C ratio of at least 0.5 .

II. Moderate-to-severe hyperchromasia.

Additionally, one of the following two features must be present:

I. Markedly, irregular nuclear borders.

II. Coarse, clumpy chromatin.

The quantity of cells is not important; a single cell meeting the above criteria can qualify for an SHGUC diagnosis. ${ }^{8}$

\section{Low grade urothelial neoplasia, (LGUN)}

LGUN includes all low-grade urothelial neoplasms of the urinary tract, such as low-grade urothelial carcinoma and papillary urothelial neoplasm of uncertain malignant potential. Under most circumstances, atypical features not associated with HGUC can be classified as benign (NHGUC) without concern for 'missing'a lowgrade neoplasm. However, if true papillary fragments are seen in a specimen (thus indicating the presence of a papillary neoplasm) and features of HGUC are absent, TPS allows for a diagnosis of LGUN to be made. ${ }^{9}$

\section{High grade urothelial carcinoma (HGUC)}

The number of atypical urothelial cells is an important criterion to classify urine cytology specimens into the 'positive' or the 'suspicious'/AUC-H categories. A cut off number of $>10$ cells to render a definitive diagnosis of HGUC seems valid from the clinical standpoint. ${ }^{10}$

\section{Other malignancies, primary and metastatic}

Primary bladder malignancies other than of urothelial origin (nonurothelial carcinoma) comprise less than $5 \%$ of all bladder tumors and include squamous cell carcinoma, adenocarcinoma, small cell carcinoma, leiomyosarcoma, angiosarcoma, melanoma, hematologic malignancy etc. Their cytology features are similar as in other sites. Secondary carcinomas are mostly direct invasion from surrounding organs as prostate, cervix, uterus, gastrointestinal tract or metastasis from distant sites like melanoma, carcinomas from kidney, breast, stomach, lung etc. ${ }^{5}$

\section{Conclusion}

The intention of The Paris Working Group is to provide a standardized guideline for reporting urine cytology. Hopefully The Paris System will be universally accepted by the cytopathologists. The Working Group with its ongoing studies will provide further evidence based information in future especially on relative risk and management protocol in each category as well as on ancillary tests.

\section{Acknowledgements}

None.

\section{Conflict of interest}

The author declares $\mathrm{n}$ conflict of interest.

\section{References}

1. Ali SZ, Leteurtre E. The official nomenclature and terminologies in diagnostic cytopathology: history, evolution, applicability and future. Ann Pathol. 2012;32(6):389-393.

2. Rosenthal DL, Wojcik E, Kurtycz DF. The Paris System for Reporting Urinary Cytology. 1st ed. USA: Springer; 2015

3. Chandra A. The Paris System for Reporting Urinary Cytopathology. Advances in bladder and prostatic tumors, Egyptian society of pathology conference, Cairo, Egypt: Springer; 2016.

4. Paris System for Reporting Urinary Cytopathology. 2015.

5. Ghosh A. The Paris System - A new insight into reporting urine cytology. Journal of Pathology of Nepal. 2016;6(11):953-958.

6. Olson MT, Barkan GA, Courtade Saïdi M, et al. Adequacy of Urine Specimens (Adequacy). In: Rosenthal, Dorothy L, et al. editors. The Paris System for Reporting Urinary Cytology, USA: Springer; 2015. p. 5-11.

7. Vanden Bussche CJ, Rosenthal DL, Olson MT. Adequacy in voided urine cytology specimens: the role of volume and a repeat void upon predictive values for high-grade urothelial carcinoma. Cancer Cytopathol. 2016;124(3):174180.

8. Vanden Bussche CJ. A review of the Paris system for reporting urinary cytology. Cytopathology. 2016;27(3):153-156.

9. Onur I, Rosenthal DL, VandenBussche CJ. Atypical urothelial tissue fragments in non instrumented voided urine specimens are associated with low but significantly higher rates of urothelial neoplasia than benign-appearing urothelial tissue fragments. Cancer Cytopathol. 2015;123(3):186-192.

10. Brimo F, Xu B, Kassouf W, et al. Urine cytology: does the number of atypical urothelial cells matter? A qualitative and quantitative study of 112 cases. Journal of the American Society of Cytopathology. 2015;4(4):232-238. 